\title{
The -308G/A Polymorphism of the Tumor Necrosis Factor-alpha Gene Is Associated with the Risk of Upper Aerodigestive Tract Cancer: A Meta-analysis
}

\author{
Jiayi Wang, ${ }^{1 *}$ Xin Jin, ${ }^{1, *}$ Hui Wang, ${ }^{2}$ Jiantang Yang, ${ }^{3}$ Lili Wang, ${ }^{1}$ Lei Lei, ${ }^{1}$ \\ Xiaoxu Li, ${ }^{1}$ Yu Zhou, ${ }^{1}$ Xin Zeng, ${ }^{1}$ Lu Jiang, ${ }^{1}$ Ga Liao, ${ }^{1}$ Hongxia Dan, ${ }^{1}$ and \\ Qianming Chen ${ }^{1}$ \\ ${ }^{1}$ State Key Laboratory of Oral Diseases, West China Hospital of Stomatology, Sichuan University, Chengdu, \\ Sichuan, P.R. China \\ ${ }^{2}$ Department of Oral Medicine, School of Stomatology, Capital Medical University, Beijing, P.R. China \\ ${ }^{3}$ Stomatological Hospital, Zunyi Medical College, Zunyi, Guizhou, P.R. China
}

Tumor necrosis factor-alpha (TNF- $\alpha$ ) has been proposed to contribute to the development of upper aerodigestive tract (UADT) cancer that is characterized by poor prognosis. The G-to-A nucleotide change at -308 of the TNF- $\alpha$ gene (-308G/A polymorphism) can increase the expression level of TNF- $\alpha$ and thus may affect the genetic susceptibility of UADT cancer. The association between the -308G/A polymorphism and UADT cancer has been widely studied, but the results published are quite controversial. To obtain a more precise conclusion, we performed a meta-analysis including 1,751 patients and 3,345 controls. The results indicated that the AA genotype of the -308G/A polymorphism had a 54\%-increased risk of UADT cancer, compared with the $G$ carriers (GG and GA genotypes) [odds ratio $(O R)=1.54,95 \%$ confidence interval $(\mathrm{Cl})$ : 1.07-2.21]. After stratified by ethnicity, the $A A$ genotype was associated with increased risk of UADT cancers in South Asians (OR $=33.18$ and $95 \% \mathrm{Cl}: 1.92-573.62$ for $A A$ vs. GA+GG) but not in Caucasians or East Asians. After stratified by tumor site, the $-308 \mathrm{G} / \mathrm{A}$ polymorphism was associated with increased risks of oropharynx cancer $(\mathrm{OR}=2.68$ and $95 \% \mathrm{Cl}: 1.34-5.35$ for $A A$ vs. $G A+G G)$ but not associated with esophagus or larynx cancer. After stratified by histological type, the $-308 \mathrm{G} / \mathrm{A}$ polymorphism was associated with increased risks of squamous cell carcinoma $(\mathrm{OR}=1.81$ and $95 \% \mathrm{Cl}: 1.15-2.84$ for $\mathrm{AA}$ vs. $G A+G G$ ) but not associated with adenocarcinoma. Our results indicate that the $-308 \mathrm{G} / \mathrm{A}$ polymorphism might contribute to an increased risk of UADT cancer susceptibility.

Keywords: epidemiology; gene polymorphism; meta-analysis; tumor necrosis factor-alpha; upper aerodigestive tract cancer

Tohoku J. Exp. Med., 2013 April, 229 (4), 245-254. ㄷ 2013 Tohoku University Medical Press

\section{Introduction}

Cancers of the upper aerodigestive tract (UADT), including malignant neoplasms of the oral cavity, pharynx, larynx, and esophagus, account for approximately $5 \%$ of all new cancer cases worldwide (Ferlay et al. 2010). UADT cancers usually have poor prognosis, and they are often accompanied with severe functional defect after treatment. Many risk factors are involved in the development of UADT cancers. Among them, tobacco, areca quid and alcohol are supposed to be the most important ones (Ansary-Moghaddam et al. 2009). It has been proved that exposing to these factors would increase the risk of the disease; however, not all individuals exposed to these factors would develop UADT cancers. This indicates that genetic differences may also contribute to the pathogenesis of UADT cancers. Therefore, a large number of studies have been carried out to explore the association of gene polymorphisms with UADT cancers (Lazarus and Park 2000; Yu et al. 2012), and many susceptibility genes have been identified, such as hypoxia-inducible factor-1 alpha (MeraMenendez et al. 2012) and microsomal epoxide hydrolase (Li et al. 2011).

Tumor necrosis factor-alpha (TNF- $\alpha$ ) was initially identified as a serum factor which could induce necrosis of transplanted tumors in mice (Carswell et al. 1975), and was found to have antitumor effects in some clinical studies (Gutman et al. 1997). However, some studies also illus-

Received September 11, 2012; revised and accepted February 28, 2013. Published online April 17, 2013; doi: 10.1620/tjem.229.245.

*These authors have equally contributed to this work.

Correspondence: Ga Liao and Hongxia Dan, State Key Laboratory of Oral Diseases, West China Hospital of Stomatology, Sichuan University, Chengdu, P.R. China.

e-mail: liaoga@gmail.com (Ga Liao), hxdan@foxmail.com (Hongxia Dan). 
trated that TNF- $\alpha$ might promote the development and spreading of cancer (Ardizzoia et al. 1992). TNF- $\alpha$ expression is mostly regulated at transcriptional level. The TNF- $\alpha$ gene is located within the MHC region on chromosome 6p21.3 (Noguchi et al. 2002). Several single-nucleotide polymorphisms have been identified in this region, and the $-308 \mathrm{G} / \mathrm{A}$ polymorphism of the TNF- $\alpha$ gene is the most frequently studied one (Guo et al. 2005; Whiteman et al. 2010; Yang et al. 2011b). It has been illustrated that the $\mathrm{G}$ to $\mathrm{A}$ nucleotide change at -308 alters the transcription factor binding site, resulting in increased production of TNF- $\alpha$ (Abraham and Kroeger 1999; Elahi et al. 2009). Numerous studies have been carried out to investigate the association of this polymorphism with UADT cancer risk (Chiu et al. 2001; Chen et al. 2005; Guo et al. 2005; Liu et al. 2005; Gupta et al. 2008; Canova et al. 2009; Yapijakis et al. 2009; Oh et al. 2010; Whiteman et al. 2010; Yang et al. 2011a); however, the results of these studies remain controversial rather than conclusive. This might partly result from the small sample size of each study. In order to get a more precise conclusion of the relationship between the $-308 \mathrm{G} / \mathrm{A}$ polymorphism and UADT cancer susceptibility, we performed a comprehensive meta-analysis of all eligible casecontrol studies.

\section{Materials and Methods \\ Publication search}

A comprehensive literature search in PubMed, EMBASE and China National Knowledge Infrastructure (CNKI) was carried out by two reviewers independently, to identify articles that had investigated the association of UADT cancer risk with TNF- $\alpha$ polymorphisms. The last updated search was performed on September 1, 2012. The search terms used were as follows: (oral or pharyngeal or pharynx or oropharyngeal or larynx or esophagus) and (cancer or carcinoma) and (Tumor Necrosis Factor or TNF or tumor necrosis factor- $\alpha$ or TNF- $\alpha$ ) and (Polymorphism or mutation or variant). Manual search of the references of original articles or review articles was performed to identify additional articles on this topic. There was no language restriction. Inclusion criteria of this meta-analysis were defined as follows: (1) evaluation of the polymorphisms of $-308 \mathrm{G} / \mathrm{A}$ and UADT cancer risk; (2) use of a case-control design based on unrelated individuals; (3) sufficient genotype distributions for cases and controls so that an odds ratio (OR) with $95 \%$ confidence interval (CI) could be estimated; (4) genotype distribution of control population should be consistent with Hardy-Weinberg equilibrium (HWE). If more than one article were published using the same case series, studies with the largest sample size were included.

\section{Data extraction}

All the identified studies had been checked by two independent reviewers and a consensus on all items had been reached. The following items were extracted using a standardized data form: first author, publication year, ethnicity, definition of cases, source of controls, genotyping method, tumor site, tumor histology, total number of cases and controls, as well as genotype distributions in cases and controls.

\section{Statistical Analysis}

Hardy-Weinberg equilibrium (HWE) in control group was firstly tested using Person's $\chi^{2}$ test. The strength of association of -308G/A polymorphism with UADT cancer risk was measured by OR and $95 \%$ CI. The statistical significance of summary OR was determined using Z-test. The pooled ORs were estimated for recessive model (AA vs. GA+GG) and dominant model (AA+GA vs. GG). The ORs of variant genotype AA compared with wild-type GG homozygote (AA vs. GG), allele A vs. allele G and GA vs. GG were also evaluated.

Heterogeneity was evaluated with a $\chi^{2}$-based Q statistic, and statistical significance was assumed for $P$ value less than 0.10 . When $P$ value was more than 0.10 , OR was pooled using the fixed-effect model, otherwise the random effect model was used. The statistical significance of $\mathrm{OR}$ was analyzed using $\mathrm{Z}$ test, $P<0.05$ was considered as statistically significant. To evaluate the ethnicity-specific, cancer-site specific and cancer-histology specific effects, subgroup analyses were performed according to ethnicity, tumor site and tumor histology. Sensitivity analyses were carried out by sequentially excluding individual studies to access the stability of the results. The possible publication bias was visually examined using the Begg's funnel plot and the degree of asymmetry was tested by Egger's test (Egger et al. 1997). All statistical tests were performed using STATA11.0.

\section{Results}

\section{Study characteristics}

The process of selecting studies was outlined in Fig. 1. After searching PubMed, Embase and CNKI electronic database, 276 articles were identified. After reading the titles and abstracts, 259 articles were excluded for no relevance to TNF- $\alpha$ gene and UADT cancer risk. After reading full texts, 6 articles were excluded for no relevance to the $-308 \mathrm{G} / \mathrm{A}$ polymorphism. One article was excluded for data overlapping. Three case-control studies were excluded for that the distribution of different genotypes in control group was not consistent with HWE. Finally, 12 case-control studies in 7 articles were identified (Chiu et al. 2001; Chen et al. 2005; Liu et al. 2005; Gupta et al. 2008; Oh et al. 2010; Whiteman et al. 2010; Yang et al. 2011a). In total, these studies included 1,751 cases and 3,345 controls. There were 5 studies in East Asian population, 1 study in South Asian population, 2 studies in Caucasian population, and 4 studies in mixed populations. Six of them investigated oropharynx cancer, 5 of them investigated esophagus cancer and 1 investigated larynx cancer. Nine studies investigated squamous cell carcinoma, 2 study investigated adenocarcinoma and 1 study investigated both cancer types. The characteristics of included case-controls were listed in Table 1. Genotype and allele distributions of each casecontrol study are listed in Table 2.

\section{Main analysis}

As shown in Fig. 2, the heterogeneity of AA vs. GA + GG for all 12 studies was assessed and the P value was 0.47 , suggesting the absence of heterogeneity. Thus, fixedeffects model was used to obtain the data. Overall OR was 


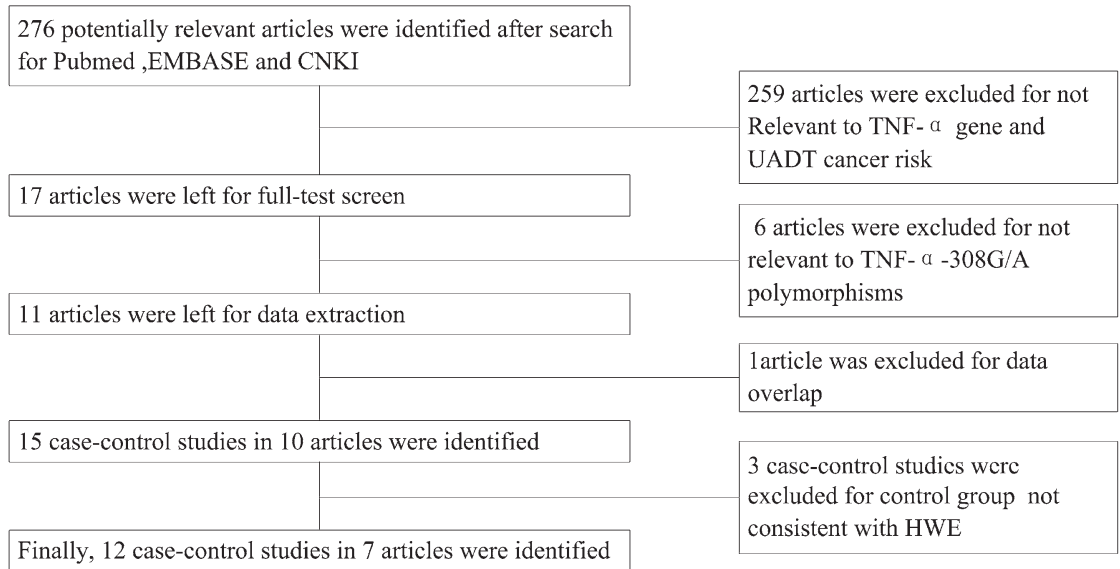

Fig. 1. Flow diagram of included/excluded studies.

Table 1. Characteristics of the 12 case-control studies included in the meta-analysis.

\begin{tabular}{|c|c|c|c|c|c|c|c|c|}
\hline Studies & Country & Ethnicity & $\begin{array}{l}\text { Cancer } \\
\text { Site }\end{array}$ & $\begin{array}{l}\text { Cancer } \\
\text { Histology }\end{array}$ & $\begin{array}{l}\text { Cases/ } \\
\text { Controls }\end{array}$ & $\begin{array}{l}\text { Case } \\
\text { gender } \\
(\mathrm{M} / \mathrm{F})\end{array}$ & Case age & Exposure factors \\
\hline Chiu CJ 2001 & China & East Asian & Oropharynx & $\mathrm{SCC}$ & $102 / 284$ & $102 / 0$ & $30-60$ & Areca \\
\hline Liu CJ 2005 & China & East Asian & Oropharynx & $\mathrm{SCC}$ & $192 / 146$ & $173 / 19$ & $54.1 \pm 9.8$ & Unknown \\
\hline Chen WC 2005 & China & East Asian & Oropharynx & $\mathrm{SCC}$ & $137 / 102$ & $125 / 12$ & $53.3 \pm 12.3$ & Toabacco \\
\hline Gupta R 2008 & India & South Asian & Oropharynx & SCC & $94 / 133$ & $76 / 18$ & $28-72$ & Toabacco \\
\hline Oh SS 2010 (E,M) & China & East Asian & Esophagus & Mixed & $194 / 340$ & $129 / 65$ & $\geq 20$ & Tobacco Alcohol \\
\hline Oh SS $2010(\mathrm{O}, \mathrm{S})$ & USA & Mixed & Oropharynx & $\mathrm{SCC}$ & $214 / 849$ & $183 / 31$ & $18-65$ & $\begin{array}{l}\text { Tobacco Alcohol } \\
\text { Marijuana }\end{array}$ \\
\hline Oh SS $2010(\mathrm{~L}, \mathrm{~S})$ & USA & Mixed & Larynx & $\mathrm{SCC}$ & $67 / 849$ & $54 / 13$ & $18-65$ & $\begin{array}{l}\text { Tobacco Alcohol } \\
\text { Marijuana }\end{array}$ \\
\hline Oh SS 2010 (E,S) & USA & Mixed & Esophagus & $\mathrm{SCC}$ & $27 / 849$ & $23 / 4$ & $18-65$ & $\begin{array}{l}\text { Tobacco Alcohol } \\
\text { Marijuana }\end{array}$ \\
\hline Oh SS 2010 (E,A) & USA & Mixed & Esophagus & $\mathrm{ACC}$ & $59 / 849$ & $48 / 11$ & $18-65$ & $\begin{array}{l}\text { Tobacco Alcohol } \\
\text { Marijuana }\end{array}$ \\
\hline Whiteman DC 2010 (E,A) & Australia & Caucasian & Esophagus & $\mathrm{ACC}$ & $253 / 1,293$ & $232 / 21$ & $18-79$ & Alcohol \\
\hline Whiteman DC 2010 (E,S) & Australia & Caucasian & Esophagus & $\mathrm{SCC}$ & $207 / 1,293$ & $120 / 87$ & $18-79$ & Alcohol \\
\hline Yang CM 2011 & China & East Asian & Oropharynx & SCC & $205 / 198$ & $205 / 0$ & $49.3 \pm 8.0$ & Areca \\
\hline
\end{tabular}

SCC, squamous cell carcinoma; ACC, adenocarcinoma; PCR-RFLP, polymerase chain reaction-restriction fragment length polymorphisms; (E,M), esophageal cancer of mixed histological types; $(\mathrm{O}, \mathrm{S})$, squamous cell carcinoma of the oropharynx; (L,S), squamous cell carcinoma of the larynx; (E,S), squamous cell carcinoma of the esophagus; (E,A), adenocarcinoma of the esophagus.

$1.54(95 \% \mathrm{CI}=1.07-2.21)$, and the test for overall effect $\mathrm{Z}$ value was $2.34(P=0.02)$ for AA vs. $\mathrm{GA}+\mathrm{GG}$ model. The results suggested that the AA genotype of the $-308 \mathrm{G} / \mathrm{A}$ polymorphism had a $54 \%$-increased risk of UADT cancer, compared with the G carriers (GG and GA genotypes). Summary of the results of other genetic comparisons are listed in Table 3.

\section{Subgroup analyses}

Subgroup analyses were performed according to ethnicity, tumor site and tumor histology. For ethnicity (Fig. 3), all the studies were divided into 4 subgroups: East Asian, South Asian, Caucasian and mixed (mixed with different populations). In recessive model (AA vs. GA $+\mathrm{GG}$ ), significantly increased risks were found among South Asian $(\mathrm{OR}=33.18,95 \% \mathrm{CI}=1.92-573.62, P=0.02)$ but not in East Asian ( $\mathrm{OR}=1.10,95 \% \mathrm{CI}=0.33-3.73)$, Caucasian (OR $=1.18,95 \% \mathrm{CI}=0.72-1.92, P=0.52)$ or Mixed group (OR $=1.60,95 \% \mathrm{CI}=0.76-3.35$ ). In the subgroup analysis of tumor site (Fig. 4), significantly increased risks were found in oropharynx cancer $(\mathrm{OR}=2.68,95 \% \mathrm{CI}=1.34-5.35, P=$ 0.01 ); no association was found with esophagus cancer (OR $=1.12,95 \% \mathrm{CI}=0.70-1.79, P=0.64)$, or larynx cancer $(\mathrm{OR}$ $=3.01,95 \% \mathrm{CI}=0.84-10.85, P=0.09)$. In the subgroup of cancer histology type (Fig. 5), significantly increased risks were found in squamous cell carcinoma $(\mathrm{OR}=1.81,95 \% \mathrm{CI}$ $=1.15-2.84, P=0.01$ ); no association was found in other cancer histology type: adenocarcinoma $(\mathrm{OR}=1.21,95 \% \mathrm{CI}$ 
J. Wang et al.

Table 2. Distribution of TNF- $\alpha$ genotype and allele among UADT cancer patients and controls.

\begin{tabular}{|c|c|c|c|c|c|c|c|}
\hline \multirow{2}{*}{ Studies } & \multicolumn{3}{|c|}{ UATD cancer } & \multicolumn{3}{|c|}{ Control } & \multirow[b]{2}{*}{ HWE } \\
\hline & GG & GA & AA & GG & GA & AA & \\
\hline Chiu CJ 2001 & 86 & 15 & 1 & 232 & 50 & 2 & 0.70 \\
\hline Liu CJ 2005 & 175 & 16 & 1 & 120 & 24 & 2 & 0.53 \\
\hline Chen WC 2005 & 125 & 12 & 0 & 88 & 14 & 0 & 0.46 \\
\hline Gupta R 2008 & 61 & 23 & 10 & 114 & 19 & 0 & 0.38 \\
\hline Oh SS 2010 (E,M) & 167 & 27 & 0 & 294 & 45 & 1 & 0.60 \\
\hline Oh SS $2010(\mathrm{O}, \mathrm{S})$ & 160 & 49 & 5 & 641 & 195 & 13 & 0.68 \\
\hline Oh SS $2010(\mathrm{~L}, \mathrm{~S})$ & 55 & 9 & 3 & 641 & 195 & 13 & 0.68 \\
\hline Oh SS $2010(\mathrm{E}, \mathrm{S})$ & 19 & 8 & 0 & 641 & 195 & 13 & 0.68 \\
\hline Oh SS 2010 (E,A) & 52 & 7 & 0 & 641 & 195 & 13 & 0.68 \\
\hline Whiteman DC 2010 (E,A) & 157 & 84 & 12 & 842 & 403 & 48 & 0.98 \\
\hline Whiteman DC 2010 (E,S) & 128 & 71 & 8 & 842 & 403 & 48 & 0.98 \\
\hline Yang CM 2011 & 180 & 23 & 2 & 155 & 43 & 0 & 0.09 \\
\hline
\end{tabular}

HWE, Hardy-Weinberg equilibrium; (E,M), esophageal cancer of mixed histological types; $(\mathrm{O}, \mathrm{S})$, squamous cell carcinoma of the oropharynx; (L,S), squamous cell carcinoma of the larynx; (E,S), squamous cell carcinoma of the esophagus; (E,A), adenocarcinoma of the esophagus.

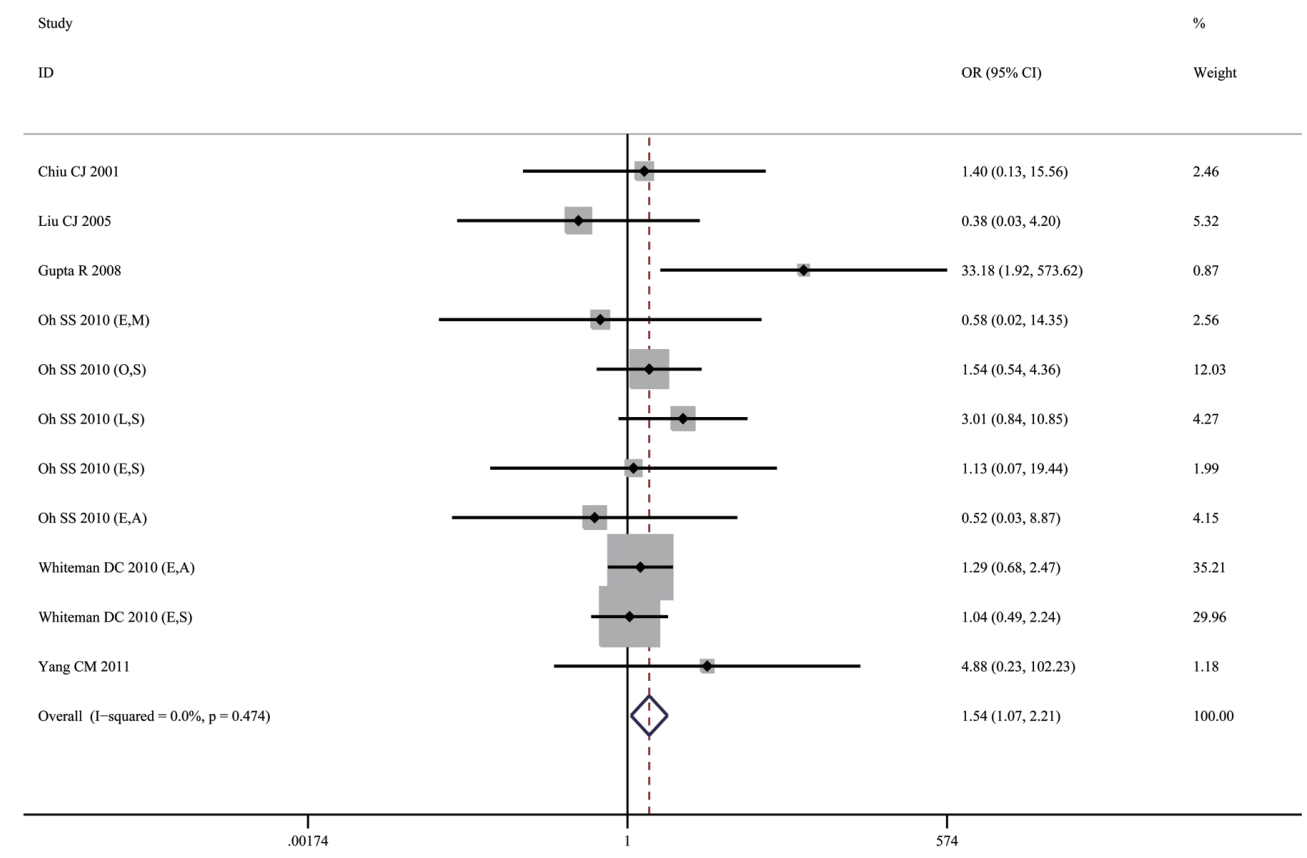

Fig. 2. Forest plot of UADT cancer risk associated with the $-308 \mathrm{G} / \mathrm{A}$ polymorphism of the TNF- $\alpha$ gene in overall analysis (AA vs. GA+GG).

The squares and horizontal lines correspond to the study-specific OR and $95 \%$ CI. The area of the squares reflects the study-specific weight. The diamond represents the summary OR and $95 \%$ CI.

$=0.65-2.27, P=0.55)$ or mixed $(\mathrm{OR}=0.58,95 \% \mathrm{CI}=0.02$ $14.35, P=0.74)$. Other genetic comparisons are summarized in Table 3.

\section{Gene-environment interaction}

Cases and controls in two studies which investigated oropharynx cancer were all areca chewers. Compared with the GG homozygote group, the AA/AG genotypes were associated with a statistically significant decreased risk of cancer risk $(\mathrm{OR}=0.63,95 \% \mathrm{CI}=0.42-0.94)($ Table 3$)$.

\section{Publication bias}

Publication bias of literature was assessed using Begg's funnel plot and Egger's test. The shapes of the funnel plots did not show any evidence of obvious asymmetry (Fig. 6). Egger's test was also performed to provide statis- 


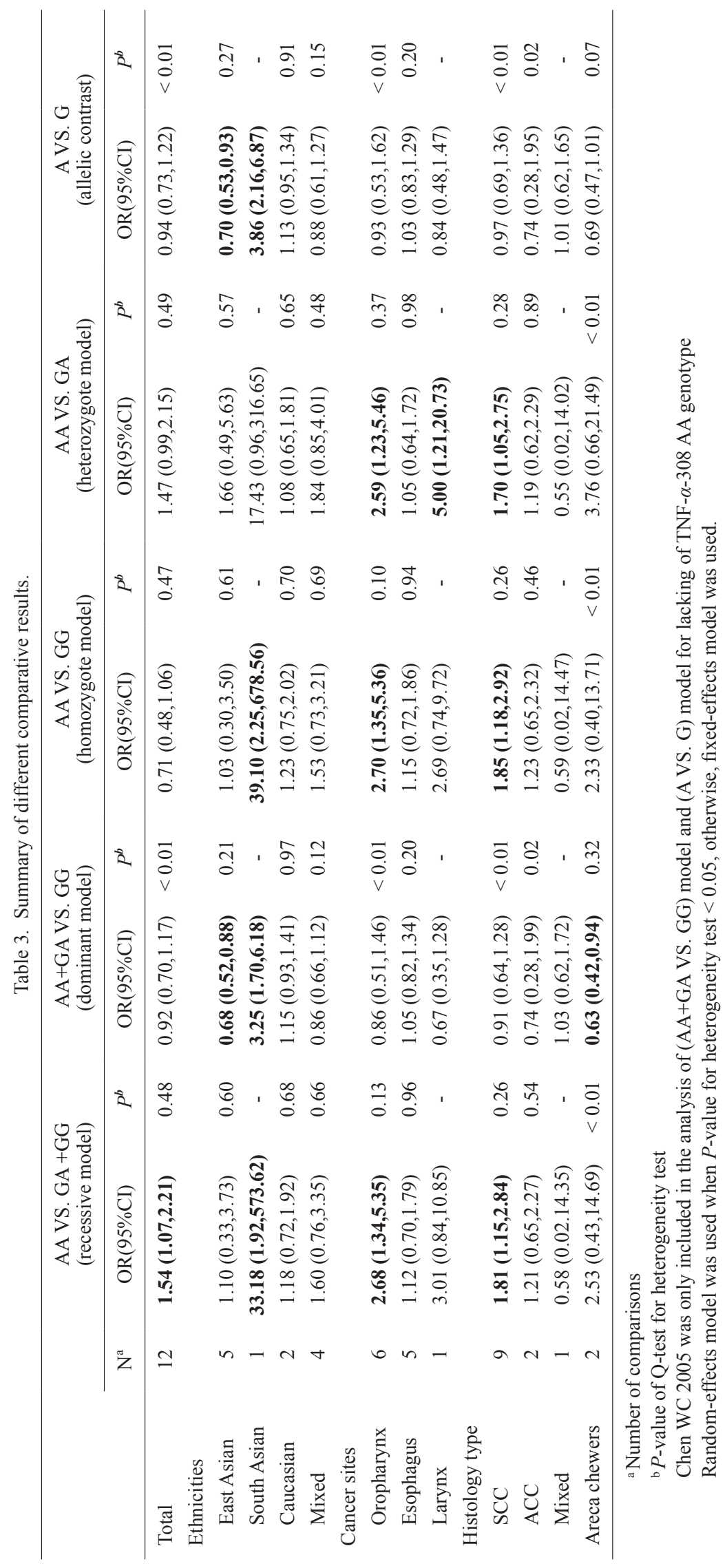




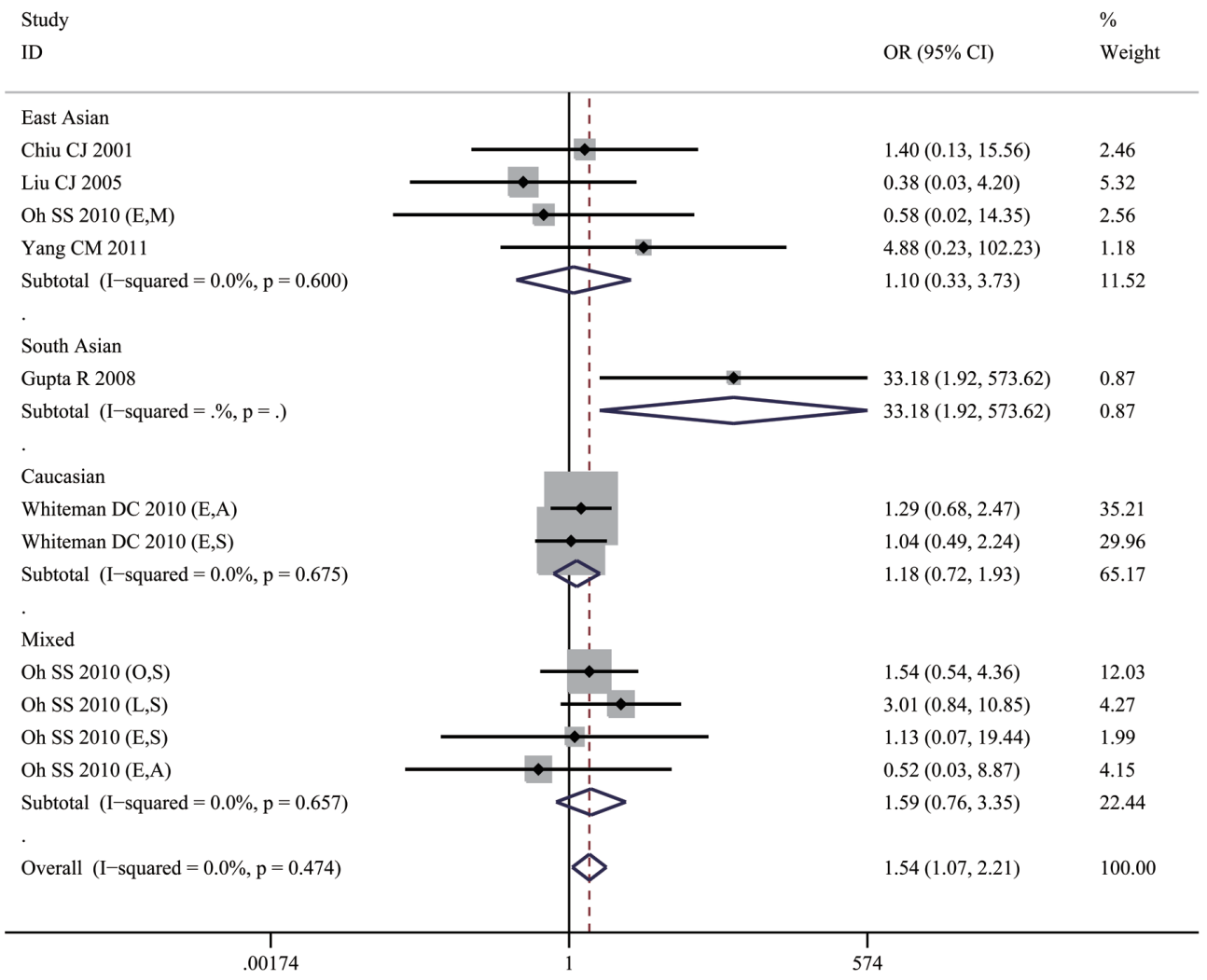

Fig. 3. Forest plot of UADT cancer risk associated with the $-308 \mathrm{G} / \mathrm{A}$ polymorphism (AA vs. GA+GG), stratified by ethnicity.

The squares and horizontal lines correspond to the study-specific OR and $95 \%$ CI. The area of the squares reflects the study-specific weight. The diamond represents the summary OR and $95 \% \mathrm{CI}$.

\begin{tabular}{|c|c|c|}
\hline $\begin{array}{l}\text { Study } \\
\text { ID }\end{array}$ & OR $(95 \% \mathrm{CI})$ & $\begin{array}{l}\% \\
\text { Weight }\end{array}$ \\
\hline Oropharynx & & \\
\hline Chiu CJ 2001 & $1.40(0.13,15.56)$ & 2.46 \\
\hline Liu CJ 2005 & $0.38(0.03,4.20)$ & 5.32 \\
\hline Gupta R 2008 & $33.18(1.92,573.62)$ & 0.87 \\
\hline Oh SS $2010(\mathrm{O}, \mathrm{S})$ & $1.54(0.54,4.36)$ & 12.03 \\
\hline Yang CM 2011 & $4.88(0.23,102.23)$ & 1.18 \\
\hline Subtotal $(\mathrm{I}-\mathrm{squared}=43.3 \%, \mathrm{p}=0.133)$ & $2.68(1.34,5.35)$ & 21.86 \\
\hline Esophagus & & \\
\hline Oh SS 2010 (E,M) & $0.58(0.02,14.35)$ & 2.56 \\
\hline Oh SS $2010(\mathrm{E}, \mathrm{S})$ & $1.13(0.07,19.44)$ & 1.99 \\
\hline Oh SS $2010(\mathrm{E}, \mathrm{A})$ & $0.52(0.03,8.87)$ & 4.15 \\
\hline Whiteman DC 2010 (E,A) & $1.29(0.68,2.47)$ & 35.21 \\
\hline Whiteman DC 2010 (E,S) & $1.04(0.49,2.24)$ & 29.96 \\
\hline Subtotal $(\mathrm{I}-$ squared $=0.0 \%, \mathrm{p}=0.956)$ & $1.12(0.70,1.79)$ & 73.87 \\
\hline . & & \\
\hline Larynx & & \\
\hline Oh SS $2010(\mathrm{~L}, \mathrm{~S})$ & $3.01(0.84,10.85)$ & 4.27 \\
\hline Subtotal $(\mathrm{I}-\mathrm{squared}=. \%, \mathrm{p}=)$. & $3.01(0.84,10.85)$ & 4.27 \\
\hline · & & \\
\hline Overall $(\mathrm{I}-$ squared $=0.0 \%, \mathrm{p}=0.474)$ & $1.54(1.07,2.21)$ & 100.00 \\
\hline${ }_{.00174}^{1}$ & & \\
\hline
\end{tabular}

Fig. 4. Forest plot of UADT cancer risk associated with the $-308 \mathrm{G} / \mathrm{A}$ polymorphism (AA vs. GA+GG), stratified by tumor site. The squares and horizontal lines correspond to the study-specific OR and 95\% CI. The area of the squares reflects the study-specific weight. The diamond represents the summary OR and $95 \% \mathrm{CI}$. 


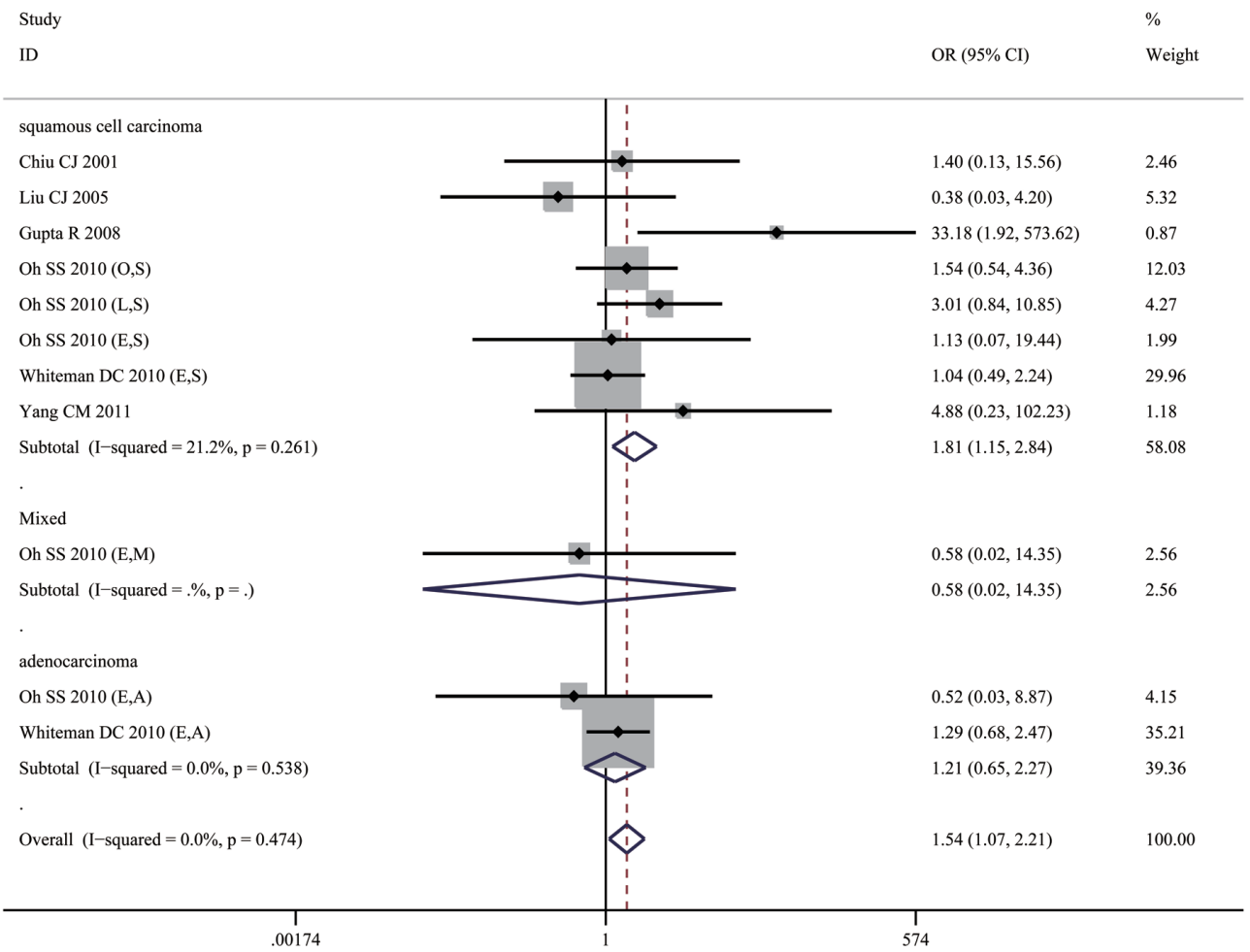

Fig. 5. Forest plot of UADT cancer risk associated with the $-308 \mathrm{G} / \mathrm{A}$ polymorphism (AA vs. GA+GG), stratified by histology type.

The squares and horizontal lines correspond to the study-specific OR and $95 \%$ CI. The area of the squares reflects the study-specific weight. The diamond represents the summary OR and $95 \%$ CI.

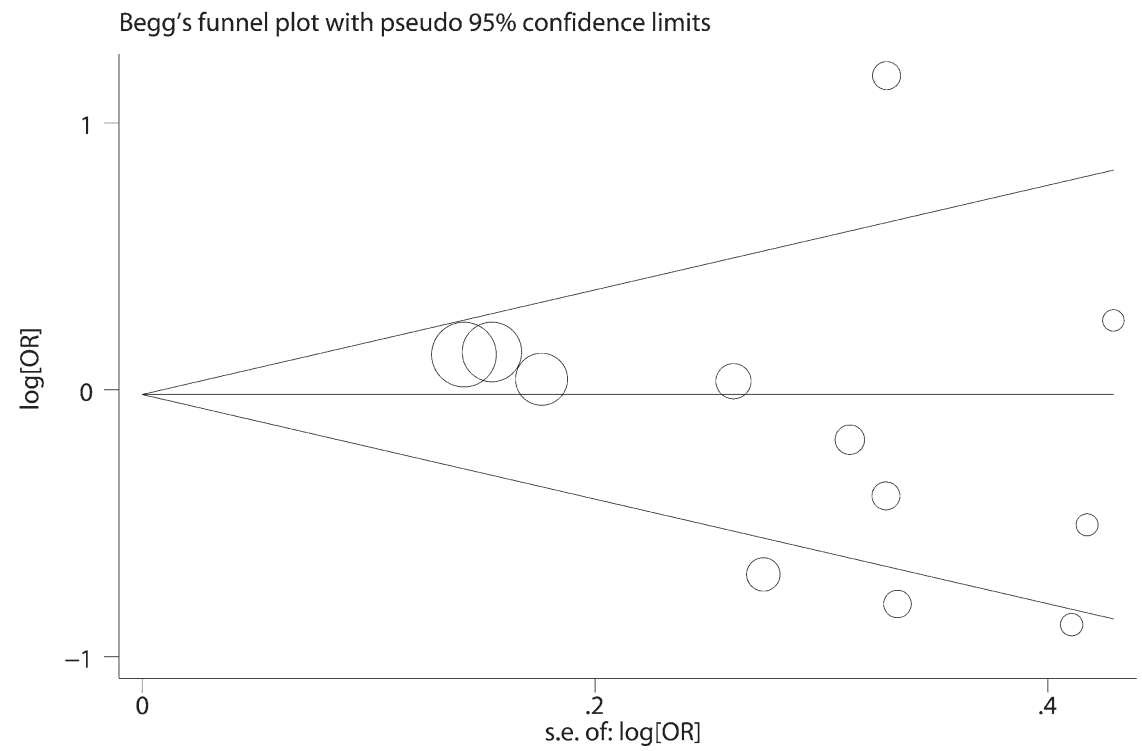

Fig. 6. Begg's funnel plot to detect publication bias test (AA vs. GA+GG).

Each point represents a separate study for the indicated association. Log (OR): natural logarithm of OR. Horizontal line represents size of effect.

tical evidence of funnel plot asymmetry. The results did not suggest any evidence of publication bias $(P=0.53)$.

\section{Sensitivity analysis}

Sensitivity analysis was performed by sequentially excluding each study to assess the stability of the current analysis. All the obtained results were similar and did not draw different conclusions. 


\section{Discussion}

UADT cancer contributes to approximately $5 \%$ of all new cancer cases worldwide (Ferlay et al. 2010). There is evidence that inflammation and cytokines play a crucial role in the development of UADT cancer (Marx 2004). TNF- $\alpha$, a critical cytokine, has been demonstrated to play an important role in several solid tumors (Balkwill 2006). Previous studies showed that the $-308 \mathrm{G} / \mathrm{A}$ polymorphism was associated with UADT cancer risk; however, the results of these studies were controversial rather than conclusive (Chiu et al. 2001; Chen et al. 2005; Liu et al. 2005; Gupta et al. 2008; Oh et al. 2010; Whiteman et al. 2010; Yang et al. 2011a). In order to confirm the association between the $-308 \mathrm{G} / \mathrm{A}$ polymorphism and the risk of UADT cancer, we performed this meta-analysis.

In the overall meta-analysis, we found that in the recessive model, overall OR for AA versus GA + GG was $1.54(95 \% \mathrm{CI}=1.07-2.21)$. TNF- $\alpha$ is a pleiotropic cytokine during the process of carcinogenesis. High doses of local TNF- $\alpha$ could suppress tumor via destructing tumor blood vessels and promoting lysis of tumor cells (Lejeune 2002). However, low doses of TNF- $\alpha$ in tumor microenvironment could promote tumor cell growth and tumor vascularization (Balkwill 2009). The reason that A allele carriers have higher UADT cancer risk might be that A allele could improve the transcription efficiency of TNF- $\alpha$, and excessive expression of TNF- $\alpha$ is associated with cancer progression as mentioned above.

After ethnicity subgroup analysis, we found a significant association between the $-308 \mathrm{G} / \mathrm{A}$ polymorphism and UADT cancer risk in South Asian, but not in East Asian, Caucasian or mixed ethnic populations. There might be several reasons for this observation. First, different ethnic populations usually have different linkage disequilibrium (LD) patterns (Frisse et al. 2001). A polymorphism locus may link with another risk variant closely in certain ethnic population. Second, environmental context which strongly varies between populations may play a role. However, as only one case-control study in South Asian population was included, the results should be taken with caution. Additional studies are needed to validate the effect of $-308 \mathrm{G} / \mathrm{A}$ polymorphism on UADT cancer risk in different ethnic groups.

After subgroup analysis according to tumor site, results showed that the $-308 \mathrm{G} / \mathrm{A}$ polymorphism was associated with an increased risk of oropharyngeal cancer; but the association was not found in esophageal and laryngeal cancer subgroups. One possible explanation is that cancers at different sites might be exposed to different environmental factors, and that the $-308 \mathrm{G} / \mathrm{A}$ polymorphism may exert varying effects in different cancers (Pelucchi et al. 2008). It should be noticed that the sample size of larynx cancer was limited which might have insufficient statistical power to detect a slight effect (Zintzaras and Lau 2008).

When stratified by tumor histology, we found that the
$-308 \mathrm{G} / \mathrm{A}$ polymorphism was associated with an increased risk of squamous cell carcinoma but the association was not found in the adenocarcinoma or the mixed group. One explanation for this discrepancy is that cancers of different histology type might have different expression profiles of TNF- $\alpha$ and thus the same polymorphism might play different roles in cancers of different origins.

Apparent gene-environment interaction was observed between the $-308 \mathrm{G} / \mathrm{A}$ polymorphism and areca chewing. Under dominant model, AA/AG carriers were associated statistically with significantly decreased risk of cancer compared with GG homozygotes. Previous studies have shown that the A allele could increase the production of TNF- $\alpha$ (Kroeger et al. 1997). TNF- $\alpha$ may induce the apoptosis process of tumor cells through binding to TNF receptors, which can lead to the activation of endonucleases and DNA fragmentation (van Horssen et al. 2006). TNF- $\alpha$ may also promote lysis of tumor cells by activating the anti-tumor $\mathrm{T}$ cell response (Xu et al. 2007). Furthermore, a previous study has shown that GG genotype could increase the risk of oral submucosa fibrosis (OSF), which is a pre-cancerous lesion with a high risk of malignant transformation (Chiu et al. 2001). Interestingly, the overall meta-analysis showed that AA genotype was associated with increased UADT cancer risk, which was contrary to the results of areca chewing subgroup. Several reasons may lead to the contradiction. First, we should notice that all of the cases and controls were East Asian in the areca chewing subgroup. Different ethnicities have different genetic background, different linkage disequilibrium patterns and different life style. These factors may interact with the $-308 \mathrm{G} / \mathrm{A}$ polymorphism and affect the results. Second, all participants in the areca chewing subgroup were male. Compared with the females, males have vital different hormone level which may interact with TNF- $\alpha$ and affect the pathogenesis of cancer (Calle and Kaaks 2004). Third, we should also notice that there were only two case-control studies in the areca chewing subgroup, which might have limited power to establish a reliable association. More case-control studies are required to validate the association.

Heterogeneity is very important in meta-analysis. There was no heterogeneity in overall comparisons in the recessive model. Even after stratified by ethnicity, tumor site and histological type of tumor, the heterogeneity was not significant in all the subgroups. Publication bias is another vital issue in meta-analysis. In this study, publication bias was assessed using Begg's funnel plots and Egger's test. No significant publication bias was found, suggesting the reliability of our results.

Some limitations of the present meta-analysis should be noticed. First, we only retrieved articles form PubMed, EMBASE and CNKI databases. It is possible that some related articles might be in some other databases and thus were not included. Secondly, although we did not find significant publication bias in this meta-analysis, it is possible that there were some unpublished results. Thirdly, data 
were not stratified by other factors, such as gender or life style as we could not extract sufficient information from the found documents. Moreover, in the stratified analysis, the participant number of some subgroups was small, which could lead to low statistic power to reveal a reliable association.

In conclusion, this meta-analysis provided evidence that the $-308 \mathrm{G} / \mathrm{A}$ polymorphism might contribute to an increased risk of UADT cancer susceptibility. Future casecontrol studies with larger sample size should be conducted to validate these findings.

\section{Acknowledgements}

This work was supported by grants from the National Natural Science Foundation of China (No. 30930100, 81072218, 30872872, 81001208, 81200791, 81061120531), International Science and Technology Cooperation Program of China (2012DFA31370), the Doctoral Program of the Ministry of Education of China (No. 20110181110055, 20120181120011) and the Specialized Research Fund for the Doctoral Program of Higher Education of China (20100181120057).

\section{Conflict of Interest}

The authors have no conflict of interest to declare.

\section{References}

Abraham, L.J. \& Kroeger, K.M. (1999) Impact of the -308 TNF promoter polymorphism on the transcriptional regulation of the TNF gene: relevance to disease. J. Leukoc. Biol., 66, 562-566.

Ansary-Moghaddam, A., Huxley, R.R., Lam, T.H. \& Woodward, M. (2009) The risk of upper aero digestive tract cancer associated with smoking, with and without concurrent alcohol consumption. Mt. Sinai J. Med., 76, 392-403.

Ardizzoia, A., Lissoni, P., Brivio, F., Tisi, E., Perego, M.S., Grassi, M.G., Pittalis, S., Crispino, S., Barni, S. \& Tancini, G. (1992) Tumor necrosis factor in solid tumors: increased blood levels in the metastatic disease. J. Biol. Regul. Homeost. Agents, 6, 103-107.

Balkwill, F. (2006) TNF-alpha in promotion and progression of cancer. Cancer Metastasis Rev., 25, 409-416.

Balkwill, F. (2009) Tumour necrosis factor and cancer. Nat. Rev. Cancer, 9, 361-371.

Calle, E.E. \& Kaaks, R. (2004) Overweight, obesity and cancer: epidemiological evidence and proposed mechanisms. Nat. Rev. Cancer, 4, 579-591.

Canova, C., Hashibe, M., Simonato, L., Nelis, M., Metspalu, A., Lagiou, P., Trichopoulos, D., Ahrens, W., Pigeot, I., Merletti, F., Richiardi, L., Talamini, R., Barzan, L., Macfarlane, G.J., Macfarlane, T.V., et al. (2009) Genetic associations of 115 polymorphisms with cancers of the upper aerodigestive tract across 10 European countries: the ARCAGE project. Cancer Res., 69, 2956-2965.

Carswell, E.A., Old, L.J., Kassel, R.L., Green, S., Fiore, N. \& Williamson, B. (1975) An endotoxin-induced serum factor that causes necrosis of tumors. Proc. Natl. Acad. Sci. USA, 72, 3666-3670

Chen, W.C., Tsai, M.H., Wan, L., Chen, W.C., Tsai, C.H. \& Tsai, F.J. (2005) CYP17 and tumor necrosis factor-alpha gene polymorphisms are associated with risk of oral cancer in Chinese patients in Taiwan. Acta Otolaryngol., 125, 96-99.

Chiu, C.J., Chiang, C.P., Chang, M.L., Chen, H.M., Hahn, L.J., Hsieh, L.L., Kuo, Y.S. \& Chen, C.J. (2001) Association between genetic polymorphism of tumor necrosis factor-alpha and risk of oral submucous fibrosis, a pre-cancerous condition of oral cancer. J. Dent. Res., 80, 2055-2059.

Egger, M., Davey, S.G., Schneider, M. \& Minder, C. (1997) Bias in meta-analysis detected by a simple, graphical test. $B M J$, 315, 629-634.

Elahi, M.M., Asotra, K., Matata, B.M. \& Mastana, S.S. (2009) Tumor necrosis factor alpha -308 gene locus promoter polymorphism: an analysis of association with health and disease. Biochim. Biophys. Acta, 1792, 163-172.

Ferlay, J., Shin, H.R., Bray, F., Forman, D., Mathers, C. \& Parkin, D.M. (2010) Estimates of worldwide burden of cancer in 2008: GLOBOCAN 2008. Int. J. Cancer, 127, 2893-2917.

Frisse, L., Hudson, R.R., Bartoszewicz, A., Wall, J.D., Donfack, J. \& Di Rienzo, A. (2001) Gene conversion and different population histories may explain the contrast between polymorphism and linkage disequilibrium levels. Am. J. Hum. Genet., 69, 831-843.

Guo, W., Wang, N., Li, Y. \& Zhang, J.H. (2005) Polymorphisms in tumor necrosis factor genes and susceptibility to esophageal squamous cell carcinoma and gastric cardiac adenocarcinoma in a population of high incidence region of North China. Chin. Med. J., 118, 1870-1878.

Gupta, R., Sharma, S.C. \& Das, S.N. (2008) Association of TNFalpha and TNFR1 promoters and 3' UTR region of TNFR2 gene polymorphisms with genetic susceptibility to tobaccorelated oral carcinoma in Asian Indians. Oral Oncol., 44, 455-463.

Gutman, M., Inbar, M., Lev-Shlush, D., Abu-Abid, S., Mozes, M., Chaitchik, S., Meller, I. \& Klausner, J.M. (1997) High dose tumor necrosis factor-alpha and melphalan administered via isolated limb perfusion for advanced limb soft tissue sarcoma results in a $>90 \%$ response rate and limb preservation. Cancer, 79, 1129-1137.

Kroeger, K.M., Carville, K.S. \& Abraham, L.J. (1997) The -308 tumor necrosis factor-alpha promoter polymorphism effects transcription. Mol. Immunol., 34, 391-399.

Lazarus, P. \& Park, J.Y. (2000) Metabolizing enzyme genotype and risk for upper aerodigestive tract cancer. Oral Oncol., 36, 421-431.

Lejeune, F.J. (2002) Clinical use of TNF revisited: improving penetration of anti-cancer agents by increasing vascular permeability. J. Clin. Invest., 110, 433-435.

Li, X., Hu, Z., Qu, X., Zhu, J., Li, L., Ring, B.Z. \& Su, L. (2011) Putative EPHX1 enzyme activity is related with risk of lung and upper aerodigestive tract cancers: a comprehensive metaanalysis. PLoS One, 6, e14749.

Liu, C.J., Wong, Y.K., Chang, K.W., Chang, H.C., Liu, H.F. \& Lee, Y.J. (2005) Tumor necrosis factor-alpha promoter polymorphism is associated with susceptibility to oral squamous cell carcinoma. J. Oral Pathol. Med., 34, 608-612.

Marx, J. (2004) Cancer research. Inflammation and cancer: the link grows stronger. Science, 306, 966-968.

Mera-Menendez, F., Hinojar-Gutierrez, A., Guijarro, R.M., de Gregorio J.G., Mera-Menendez, E., Sanchez, J.J., Quintanilla, M., Cerezo, L. \& Gamallo, C. (2012) Polymorphisms in HIF1alpha affect presence of lymph node metastasis and can influence tumor size in squamous-cell carcinoma of the glottic larynx. Clin. Transl. Oncol. [Epub ahead of print].

Noguchi, E., Yokouchi, Y., Shibasaki, M., Inudou, M., Nakahara, S., Nogami, T., Kamioka, M., Yamakawa-Kobayashi, K., Ichikawa, K., Matsui, A. \& Arinami, T. (2002) Association between TNFA polymorphism and the development of asthma in the Japanese population. Am. J. Respir. Crit. Care Med., 166, 43-46.

Oh, S.S., Chang, S.C., Cai, L., Cordon-Cardo, C., Ding, B.G., Greenland, S., He, N., Jiang, Q., Kheifets, L., Le, A., Lee, Y.C., Liu, S., Lu, M.L., Mao, J.T., Morgenstern, H., et al. (2010) Single nucleotide polymorphisms of 8 inflammationrelated genes and their associations with smoking-related 
cancers. Int. J. Cancer, 127, 2169-2182.

Pelucchi, C., Gallus, S., Garavello, W., Bosetti, C. \& La Vecchia, C. (2008) Alcohol and tobacco use, and cancer risk for upper aerodigestive tract and liver. Eur. J. Cancer Prev., 17, 340344.

van Horssen, R., Ten Hagen, T.L. \& Edgemont, A.M. (2006) TNFalpha in cancer treatment: molecular insights, antitumor effects, and clinical utility. Oncologist, 11, 397-408.

Whiteman, D.C., Parmar, P., Fahey, P., Moore, S.P., Stark, M., Zhao, Z.Z., Montgomery, G.W., Green, A.C., Hayward, N.K. \& Webb, P.M. (2010) Association of Helicobacter pylori infection with reduced risk for esophageal cancer is independent of environmental and genetic modifiers. Gastroenterology, 139, 73-83.

Xu, J., Chakrabarti, A.K., Tan, J.L., Ge, L., Gambotto, A. \& Vujanovic, N.L. (2007) Essential role of the TNF-TNFR2 cognate interaction in mouse dendritic cell-natural killer cell crosstalk. Blood, 109, 3333-3341.

Yang, C.M., Hou, Y.Y., Chiu, Y.T., Chen, H.C., Chu, S.T., Chi, C.C., Hsiao, M., Lee, C.Y., Hsieh, C.J., Lin, Y.C., Hsieh, Y.D.
\& Ger, L.P. (2011a) Interaction between tumour necrosis factor-alpha gene polymorphisms and substance use on risk of betel quid-related oral and pharyngeal squamous cell carcinoma in Taiwan. Arch. Oral Biol., 56, 1162-1169.

Yang, Y., Feng, R., Bi, S. \& Xu, Y. (2011b) TNF-alpha polymorphisms and breast cancer. Breast Cancer Res. Treat., 129, 513-519.

Yapijakis, C., Serefoglou, Z., Vylliotis, A., Nkenke, E., Derka, S., Vassiliou, S., Avgoustidis, D., Neukam, F.W., Patsouris, E. \& Vairaktaris, E. (2009) Association of polymorphisms in Tumor Necrosis Factor Alpha and Beta genes with increased risk for oral cancer. Anticancer Res., 29, 2379-2386.

Yu, H., Liu, Z., Huang, Y.J., Yin, M., Wang, L.E. \& Wei, Q. (2012) Association between Single Nucleotide Polymorphisms in ERCC4 and Risk of Squamous Cell Carcinoma of the Head and Neck. PLoS One, 7, e41853.

Zintzaras, E. \& Lau, J. (2008) Synthesis of genetic association studies for pertinent gene-disease associations requires appropriate methodological and statistical approaches. J. Clin. Epidemiol., 61, 634-645. 